\title{
Robust Markers for Blood Vessels Segmentation: A New Algorithm
}

\author{
Roberto Rodríguez, Teresa E. Alarcón, and Oriana Pacheco \\ Institute of Cybernetics, Mathematics and Physics (ICIMAF) \\ Digital Signal Processing Group \\ Calle 15 No. 551 e/ C y D CP 10400, La Habana, CUBA \\ rrm@.icmf.inf.cu, tere@icmf.inf.cu
}

\begin{abstract}
In this paper, we present a new algorithm to obtain robust markers for blood vessels segmentation in malignant tumors. We propose a two-stage segmentation strategy which involves: 1) extracting an approximate region containing blood vessels and part of the background, and 2) segmenting blood vessels from the background within this region. The approach was effectively very useful in blood vessels segmentation and its validity was tested by using the watershed method. The proposed segmentation technique is tested on manual segmentation. It is demonstrated by extensive experimentation, by using real images, that the proposed strategy was suitable for our application.
\end{abstract}

\section{Introduction}

Information contained within sampled medical image data sets is essential to several clinical tasks. Advances in technology allowed clinician not only the visualization, but also the identification of different objects. A major hurdle in the effective use of this technology is the image segmentation, where pixels are grouped into regions based in image features [1].

In the study of the angiogenesis process, the pathologists analyse all information related with blood vessels by using a microscope [2-4]. This work is very tedious and time consuming and obviously, the automation of this analysis is highly desirable. In such a sense, an useful task for digital images processing should be the segmentation of blood vessels.

Segmentation and contour extraction are key points of image analysis. Many segmentation methods have been proposed for medical image data [5-7]. Unfortunately, segmentation using traditional low level image processing techniques, such as thresholding, gradient, and other classical operations, requires a considerable amount of interactive guidance in order to get satisfactory results. Automating these model free approaches is difficult because of shape complexity, shadows, and variability within and across individual objects. Furthermore, noise and other image artifacts can cause incorrect regions or boundary discontinuities in objects recovered from these methods.

The watersheds is a powerful segmentation tool developed in mathematical morphology $[8,9]$. However, the correct way to use watersheds for grayscale image segmentation consists in first detecting markers of the objects to be extracted. The design of robust marker detection techniques involves the use of knowledge specific of images under study. Not only object markers, but also background markers need to be extracted. 
The goal of this paper is to present a new algorithm to obtain robust markers for blood vessels segmentation in malignant tumors. The validity of our strategy was tested by using the watersheds method, where, according to criterion of physicians, blood vessels were contoured well.

This paper is organized as follows: In Section II is outlined the theoretical aspects. In section III, we give the features of the studied images. In section IV, we present the steps to obtain markers, we also discuss an algorithm. Finally, we describe our conclusions in Section V.

\section{Theoretical Aspects}

This section presents the most important theoretical aspects.

\subsection{Pre-processing}

With the goal of diminishing the noise in the original images we used the Gauss filter, where we carried out the process of Gaussian smoothing with $\sigma=3$ and a $3 \times 3$ window size. We carried out also the morphological opening. In this work, we used a structuring element type rhombus of $3 \times 3$ size.

\subsection{Watershed Segmentation}

Let us consider a two-dimensional grayscale picture $F$ whose definition domain is denoted $\mathrm{D}_{\mathrm{F}} \subset \mathrm{Z}^{2} . F$ is supposed to take discrete (gray) values in a given range $[0, \mathrm{~L}]$, $\mathrm{L}$ being an arbitrary positive integer. In the following, we consider grayscale images as numerical functions or as topographic relief.

Definition 1 (Regional Minimum).

A regional minimum $\mathrm{M}$ at altitude $\mathrm{h}$ of grayscale image $F$ is a connected component $\mathrm{C}$ of $\mathrm{T}_{\mathrm{h}}(F)$ such that $\mathrm{C} \cap \mathrm{T}_{\mathrm{h}-1}(F)=\phi, \mathrm{T}_{\mathrm{h}}(F)$ being a threshold of $F$ at level $\mathrm{h}$.

Definition 2 (Watershed by Immersion).

We can figure that we have pierced holes in each regional minimum of $F$, this picture being regarded as a topographic surface. We then slowly immerse this surface into a lake. Starting from the minimum of lowest altitude, the water will progressively fill up the different catchment basins of $F$. Now, at each pixel where the water coming from two different minimum would merge, we build a dam (see Fig. 1). At the end of this immersion procedure, each minimum is completely surrounded by dams, which delimit its associated catchment basin. The whole set of dams which has been built thus provides a tessellation of $F$ in its different catchment basins. These dams correspond to the watershed of $F$, that is, these represent the edges of objects.

\section{Features of the Studied Images}

The studied images were of arteries, which had atherosclerotic lesions and these were obtained from different parts of the human body, from more of 80 autopsies. In Figure 2 can be seen typical image, which were captured via MADIP system with a resolution of $512 \times 512 \times 8 \mathrm{bit} /$ pixels [10]. 


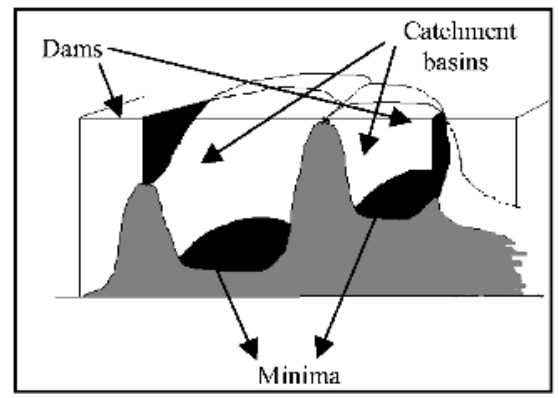

Fig. 1. Building dams at the places where the water coming from two different minimum would merge.

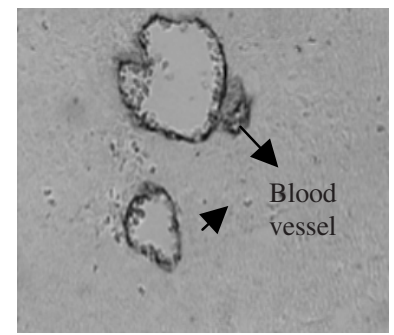

Fig. 2. These images represent the angiogenesis process. Blood vessels are marked with arrows.

There are several notable characteristics of this image, which are common to typical images that we encounter in the tissues of biopsies:

1. High local variation of intensity is observed both, within blood vessels (BV) and the background. However, the local variation of intensities is higher within BV than in background regions.

2. It is common of these images the diversity in shape and size of BV.

\section{Experimental Results: Discussion}

\subsection{Obtaining the Region of Interest}

The next stage of the strategy is to segment the approximate region, that is, a region which contains the blood vessel and its neighboring background. The exact shape and size of this region are not important, and hence the region is referred to as an approximate region. A measure of local variation of intensity is provided by the variance of the gray level intensity. The result of applying this procedure is shown in Figure 3.

Figure 3 shows that the high variance corresponds to blood vessels while that the low variance belongs to background. We verified in what we did that for large window sizes the results were poor. On the one hand, it was obtained a higher homogeneity of the region, and on the other hand blood vessels were notably fattened.

We obtained the region of interest by applying a global threshold to the local variation of intensity. Figure 4 shows the obtained result of this segmentation process.

Then, we introduced the following algorithm to obtain robust markers for blood vessels segmentation in malignant tumors. 


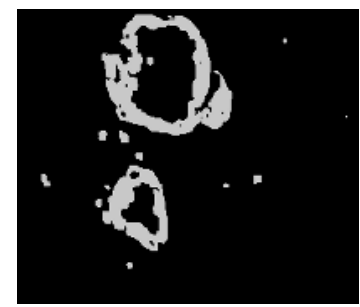

Fig. 3. Obtaining a region of interest. The variance map was obtained with $5 \times 5$ window size.
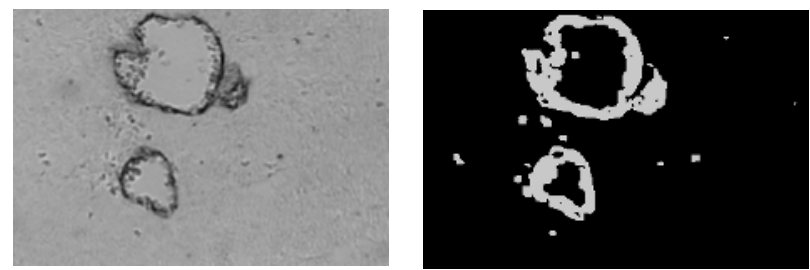

Fig. 4. (a) Original image. (b) Regions of interest.

\subsection{Algorithm to Obtain Robust Markers}

The steps of the algorithm are described below.

1. Obtain the regions of interest. Let IREZI be the resulting image.

2. Label the resulting image of the step 1 . Then, to create an auxiliary image, let IA1 be. All pixels of this image are put in zero. Scan IREZI at iterative way, then in this image all the background is labeled with value equal to 1 .

3. With the goal of finding connected components (BV), scan IREZI again from the top to the bottom and from the left to the right. If there is a pixel, which belongs to a connected component and in IAl this pixel has value zero, then other iterative method begins to work. This new iterative method marks with a determined value within IA1 all pixels belonging to a connected component. In addition, pixels within IREZI are marked also with a value, which identifies the connected component to which they belong. This action is carried out in the whole image. As this step is finished, in the IREZI image all the connected components were filled and in IA1 all the connected components were labeled.

4. Create other auxiliary image (let IA2 be) with the same values of the IA1 image. Create also an array, which controls if a connected component was reduced. In the IA2 image is where in each step the reduction of the connected components are obtained, the final result in the IA1 image is represented.

5. Scan the labeled image (IA1). When a pixel is found, which belongs to a connected component, via other iterative method the same is reduced and in the IA2 image all the frontiers of the connected component are marked. If some pixel within the connected component is yet, which is no frontier, then in IA2 and IA1 the frontiers are eliminated and this function begins again until that all points are frontiers. In this case, the obtained result (reduction) is taken as the mark. In the 
array (see step 4) is indicated that the labeled component with this value was processed and it is begun to look for other component.

6. Finish when the IA1 image is completely scanned. When this step is concluded, in the IA1 image all marks of BV are. These marks are collocated in the IREZI image, where the connected components of the IREZI image after the step two were filled. The IREZI image is the resulting image.

Figure 5 shows the obtained results of applying this algorithm. In Figure 5(b) can be seen that the mark is unique to each of blood vessels, which is always within them. Figure 6 depicts all steps of the proposed strategy to obtain robust markers in blood vessel images.

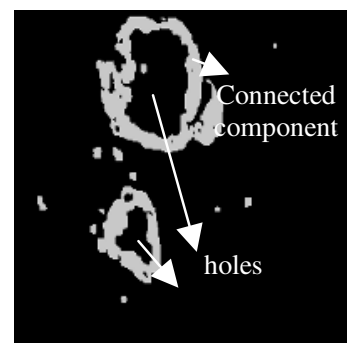

(a)

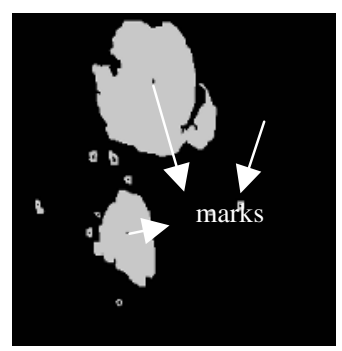

(b)

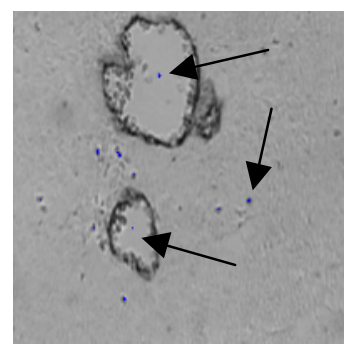

(c)

Fig. 5. (a) Image with regions of interest. The arrows indicate the connected components and the holes within them. (b) Image with the connected components filled, the interior hole is the obtained mark (c) Superimposed original image on the obtained marks, which some are indicated with arrows.

\subsection{Application of the Obtained Strategy in the Watershed Segmentation}

There are many applications in digital image processing where is very important to eliminate the minima not wished and consider only the necessary minima (see section 2). In the following, we propose a way to detect these necessary minima:

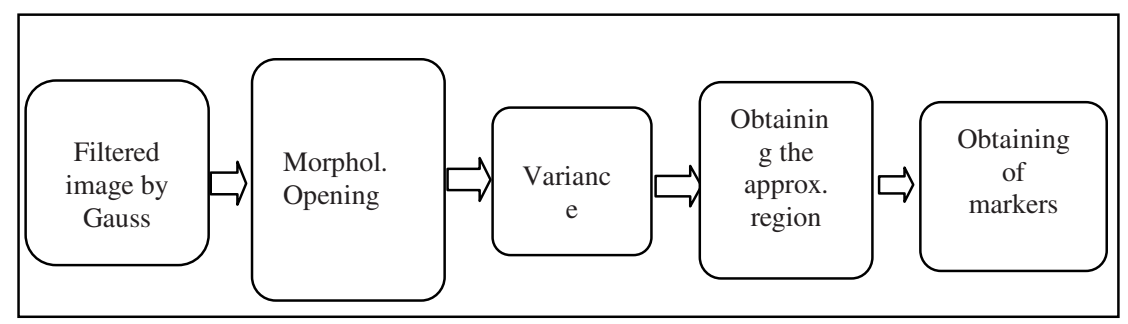

Fig. 6. Steps to obtain markers in angiogenesis images

(a) Carry out a reconstruction by geodesic dilations with a rhombus of size $5 \times 5$ (in pixels) as structuring element and with constant $\mathrm{h}=30$ [8].

(b) Carry out a morphological gradient to the resulting image of the reconstruction.

(c) Define via a horizontal profile from the gradient image the pixels belonging to background and those belonging to blood vessels. Of this way are obtained two thresholds. Then, carrying out a thresholding, in which we assigned a value to blood vessels and other to background. 
The obtained result of applying the steps (a), (b) and (c) is pictured in Figure 7. In many practical cases the watershed segmentation produces an over-segmentation if one does not obtain robust markers. In fact, when transformation is directly applied to the gradient image is produced an over-segmentation due to existing noise in the original image. Figure 8 shows this effect.

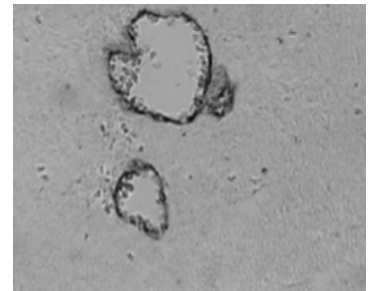

(a)

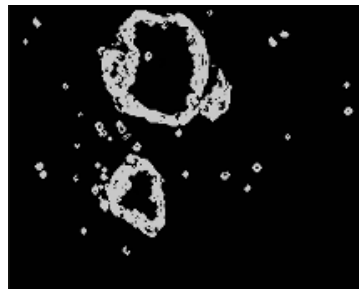

(b)

Fig. 7. (a) Original image. (b) Resulting image.

Figure 8 (b) shows the poor result when are not obtained good markers. It is evident as the edges are not correctly defined. However, in Figure 9, we show the obtained result of applying our strategy.

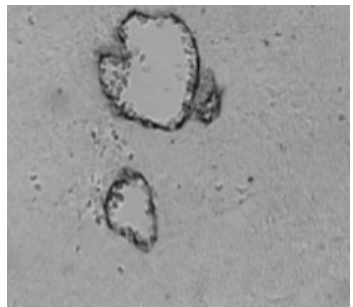

(a)

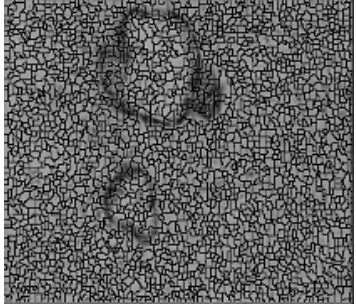

(b)

Fig. 8. (a) Original image. (b) Watershed segmentation. It is evident the over-segmentation.

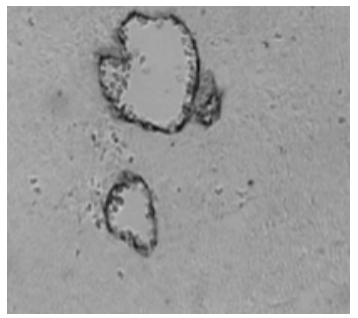

(a)

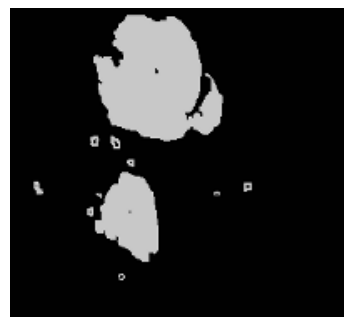

(b)

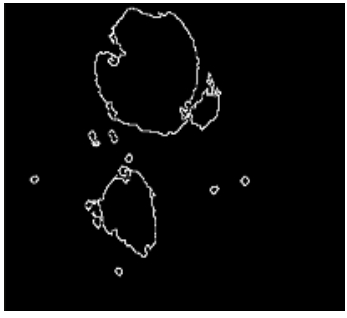

(c)

Fig. 9. (a) Original image. (b) Image of marks. (c) Watershed segmentation. It is evident the quality of the obtained result.

As can be seen in Figure 9 (c) the edges of blood vessels were correctly defined. In order to verify the good performance of our strategy, we superimposed the contours on the original image. This result can be observed in Figure 10. It is evident that were obtained the exact edges of blood vessels. In addition, the obtained edges were continuos, which is very important for this application. With the classical methods is not possible to obtain continuos edges. 


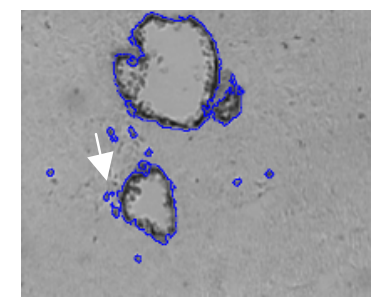

Fig. 10. Contours superimposed on the original image.

\section{Conclusions}

In this work, we presented a new strategy to obtain robust markers for blood vessel segmentation via watershed method. A such a sense, we introduced a new algorithm, which identifies correctly blood vessels and eliminate considerably all spurious information. In order to obtain such goal, we carried out a study of the region of interest. We demonstrated by extensive experimentation, by using real images data, that the proposed strategy was fast and robust for the images which were considered.

\section{References}

1. R. González and R. E. Woods, Digital Image Processing, Addison-Wesley Publishing Company, Inc., Reading, Massachusetts, E. U. A, 1992.

2. Boschner, B. H., Cote, R. J., Weidner, N., Groshen, S., Chen, S. C., Skinner, D. G., Nichols, P. W., "Angiogenesis in bladder cancer: relationship between microvessel density and tumor prognosis”, J. Natl. Cancer Inst., 87: 21, no.1, 1603-1612, 1995.

3. Díaz-Flores, L., Gutiérrez, R., Varela, H., “Angiogenesis: an update”, Histol Histopath 9: 807-843, 1992.

4. Weidner N., Carroll, P. R., Flax, J., Blumenfeld, W., Folkman, J., “ Tumor angiogenesis correlates with metastasis in invasive prostate carcinoma", American Journal of Pathology, vol. 143, no.2, 1993.

5. W. Kenong, D. Gauthier and M. D. Levine, "Live Cell Image Segmentation", IEEE Transactions on Biomedical Engineering, vol.42, no. 1, enero 1995.

6. J. Sijbers, P. Scheunders, M. Verhoye, A. Van der Linden, D. Van Dyck, E. Raman, "Watershed-based segmentation of $3 D$ MR data for volume quantization", Magnetic Resonance Imaging, vol. 15, no. 6, pp 679-688, 1997.

7. C. Chin-Hsing, J. Lee, J. Wang and C. W. Mao, "Color image segmentation for bladder cancer diagnosis", Mathl. Comput. Modeling, vol. 27, no. 2, pp. 103-120, 1998.

8. Vincent, L.: "Morphological grayscale reconstruction in Image Analysis: Applications and Efficient Algorithms". IEEE Transactions on Image Processing, vol.2, pp. 176-201, April, 1993.

9. Vicent, L and Soille, P.: "Watersheds in digital spaces: An efficient algorithm based on immersion simulations”, IEEE Transact. Pattern Anal. Machine Intell., 13:583-593; 1991.

10. R. Rodríguez, T. E. Alarcón and L. Sánchez: "MADIP: Morphometrical Analysis by Digital Image Processing", Proceedings of the IX Spanish Symposium on Pattern Recognition and Image Analysis, Vol. I, pp. 291-298, ISBN 84-8021-349-3, 2001, Spain. 